\title{
A Novel and Open-Source Application for Vasculature Dataset Analysis and Visualization
}

Jacob Bumgarner ( $\nabla$ jrbumgarner@mix.wvu.edu )

West Virginia University https://orcid.org/0000-0002-0387-9263

\section{Randy Nelson}

West Virginia University

\section{Article}

Keywords: Vasculature analysis, 3D vasculature, 2D vasculature, vasculature visualization, vascular networks

Posted Date: June 11th, 2021

DOI: https://doi.org/10.21203/rs.3.rs-608609/v1

License: (c) (1) This work is licensed under a Creative Commons Attribution 4.0 International License. Read Full License 
Submitted to Nature Methods on 10 June 2021

2

3

A Novel and Open-Source Application for Vasculature Dataset Analysis and Visualization

${ }^{*}$ To whom correspondence should be addressed: jrbumgarner@mix.wvu.edu 


\section{Abstract}

Vascular networks can dictate and indicate states of health and disease. Structural analyses of 36 these networks can facilitate improved understanding of disease states. Recent advances in preclinical 37 imaging techniques and segmentation software have led to the generation of large-scale vasculature 38 datasets. However, these advances have not been accompanied by the development of modernized, 39 open-source analysis software packages. Here we describe VesselVio, an application developed to 40 analyze and visualize pre-segmented 2D and 3D vasculature datasets. Vasculature datasets can be 41 loaded and analyzed with custom parameters to extract numerous quantitative whole-network and 42 individual segment features. Visualization of results and accuracy inspections can be conducted using 43 the interactive visualization tool. The utility and compatibility of VesselVio is demonstrated via the 44 analysis of 3D inferior colliculus segmentations from female and male mice as well as the analysis of $452 \mathrm{D}$ retinal fundus images of control, glaucomatous, and diabetic retinopathy patients.

\section{Keywords}

48 Vasculature analysis, 3D vasculature, 2D vasculature, vasculature visualization, vascular networks. 
Introduction

Microvascular networks serve vital roles in the maintenance of cellular function in all tissue by providing finely tuned routes for the delivery and clearance of nutrients and waste, signaling molecules, and trafficking immune cells. Often, vascular networks become disorganized or impaired by diseases or illnesses, such as COVID-19 ${ }^{1}$, diabetes ${ }^{2}$, stroke $^{3}$, or Alzheimer's disease ${ }^{4}$, which can lead to compounding detrimental health consequences. Accordingly, detailed characterization of the distinct structural features of these networks may enable improved comprehension and identification of underlying pathological conditions.

In recent years, high-resolution and large-scale 3D vasculature dataset generation has been facilitated by continued developments of light sheet fluorescent microscopy (LSM) ${ }^{5-7}$ and micro computed tomography imaging techniques $(\mu \mathrm{CT})^{8,9}$. Simultaneously, constant improvements in computational power and availability of powerful programming languages provide greater means by which to process and extract detailed features from the resulting datasets. Together, micron-scaled resolution imaging and powerful computational tools can be utilized to reveal intricate details of microvascular networks that will lead to an improved understanding of microvascular structure, function, and remodeling in health and disease.

However, ongoing advancements in imaging technologies and open-source image segmentation software for $3 \mathrm{D}$ vasculature datasets ${ }^{6,7,10}$ have not yet been accompanied by publication of free and easily accessible analysis software toolkits. For example, there are several freely available older analysis tools, but they are unable to extract features from 3D datasets ${ }^{11,12}$. Other modern openaccess analysis packages have similar 2D limitations ${ }^{13-15}$. Existing open-source analysis tools and software packages capable of analyzing 3D vasculature have several limitations. Several of these tools can extract 3D network characteristics, yet they depend on simple centerline analyses, provide limited feature output, and markedly over-label branchpoints, producing inaccurate results ${ }^{7,16}$. Other modern analysis packages that extract more accurate ${ }^{6,17}$ and detailed features ${ }^{18,19}$ from 3D vascular networks 
75 require experience with programming or interaction with terminals, potentially leading to unwelcome 76 usage barriers and steep learning curves for researchers. Many publications also make use of private 77 code or proprietary software for feature extractions ${ }^{20-22}$. In these instances, limited code availability and 78 high software prices can hinder widespread analysis access. Thus, there is an apparent void for a freely 79 accessible tool for 3D vasculature dataset analysis.

Here we present Vesse/Vio, an open-source application for the analysis and visualization of 2D

81 and 3D vasculature datasets. We created this application by first developing backend pipelines for 82 dataset analysis and visualization with several novel feature extraction techniques. Both backend 83 pipelines leverage high-level python libraries, just-in-time compilers, and parallel processing for rapid, 84 detailed feature extraction and complementary visualization of datasets. We then designed a stand85 alone and downloadable front-end application to wrap these pipelines with ease of use in mind. To test 86 the performance and utility of VesselVio, we analyzed ground-truth synthetic vascular datasets, and we 87 analyzed $2 \mathrm{D}$ retinography datasets and $3 \mathrm{D}$ vasculature datasets generated by $\mu \mathrm{CT}$, LSM, and MRI 88 imaging. 


\section{Results}

Centerline Extraction and Graph Theory Enable Detailed Vascular Network Feature Extraction

We sought to build an open-source application that allows users to extract numerous quantitative features from 2D and 3D vascular networks. Different imaging techniques can produce varying qualities of raw images that require unique vessel detection and thresholding processes. Because of this, 95 VesselVio was developed for vascular datasets that have already been binarized and segmented, enabling it to be used with datasets of any imaging origin (Figure 1A). This development choice enabled analysis pipeline optimization for increased accuracy and speed of feature identification. The visualization component for the program enables researchers to examine their results alongside original volume meshes for accuracy inspection.

The features of a vascular network can be extracted by identifying centerlines of the network and creating undirected graphs of the centerline points ${ }^{23,24}$. To locate vessel centerlines, a widely used 2D and 3D thinning algorithm for binarized images was employed to extract the skeleton of our datasets $^{25}$. Edge-connectivity of the skeleton points is identified and used to create an undirected graph with the resulting edges and points, $G=(V, E)$. Following initial graph construction, the datasets follow a series of correction processes to filter out spurious branchpoint labels, smooth centerlines, and remove isolated/endpoint segments at user-defined size (Figure 1B). These initial processing stages enable downstream quantifications of network and segment features (Figure 1C).

Figure 1 - Overview of the analysis and visualization pipelines of VesselVio. a, Pre-segmented datasets are loaded into the program where analyses can be conducted using custom analysis and feature export parameters. $\mathbf{b}$, Centerlines are extracted from the loaded datasets, and undirected graphs are created with the resulting vertices and edges. Centerlines are smoothed, spurious branch points are filtered, and segments are filtered prior to result extraction. c, The resulting datasets can be visualized for inspection of the accuracy and quality of results. 


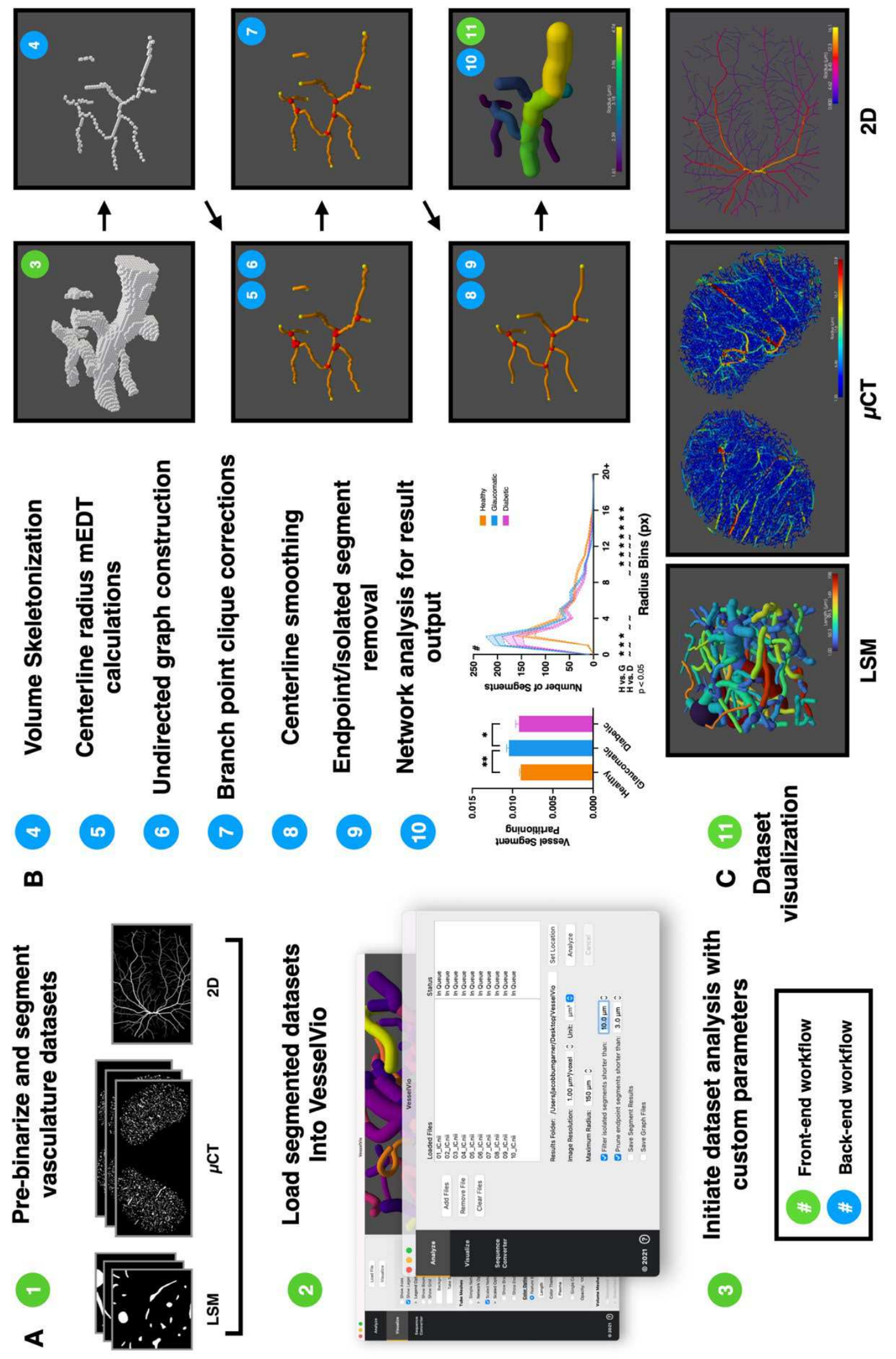




\section{Endpoints and Branchpoint Clique Filtering}

After graph construction, network endpoints and branchpoints are identified by examining the degree of connectivity of the centerline vertices (Figure 2A). Centerline neighbor 26-connectivity is a commonly used method for branchpoint and endpoint identification ${ }^{7,16,18}$. Although endpoints can be accurately detected using this technique, branchpoints are spuriously labeled and counts become artificially inflated (Figure 2B). Some programs allow for interactive user input to correct incorrectly labelled segments, centerlines, or branchpoints (e.g., Imaris ${ }^{8}$ ). However, manually correcting branchpoints in 3D datasets can become tedious and time-intensive, particularly when datasets are several gigabytes large. Because VesselVio is designed for automated analysis without user guidance, a set of algorithms were implemented to automatically filter incorrectly identified branchpoints from analyzed datasets.

Spuriously labelled branchpoints form small loops in the constructed graphs, otherwise known as cliques. Branchpoint cliques can be identified by filtering the graph of endpoints, 2-degree vertices, and single or two-component branchpoints. These cliques contain candidate branchpoints with one or more loops generated by between-edges (Figure 2A). Previous techniques used to eliminate spurious branchpoint labels rely on parent vessel orientations ${ }^{26}$ or generate candidate weights based on 26neighborhood connectivity ${ }^{27}$. Instead, VesselVio implements a series of filtering algorithms that apply branchpoint candidate weighting based on vertex radius, neighbor radius, and degree of connectivity (Supplementary Notes). Weighting candidates based on radius in addition to connectivity also mimics parent vessel hierarchy and improves calculations of segment radii.

To test our filters, a set of synthetically generated vasculature datasets with branchpoint labels were analyzed and results were compared to previous programs ${ }^{16,28}$. Labeled branchpoints from these 
datasets were considered as ground truths. VesselVio clique filtering resulted in a mean $97.5 \%$ accuracy of branchpoint labeling by removing an average of $99.6 \%$ of identified cliques in the datasets $(N=20)$ (Supplementary Notes; Supplementary Data 1$)$. The hierarchical branchpoint filtering of VesselVio with a mean of $2.5 \%$ error outperforms the mean $122.2 \%$ error of previous techniques (Figure 2B). Further, application of the filtering algorithms resulted in correction of $99.7 \%(\mathrm{~N}=10)$ and 99.9\% $(N=45)$ of the identified branchpoint cliques in the 3D and 2D datasets that are analyzed below, respectively (Supplementary Data 1).

Figure 2 - Spurious branch point filtering and modified radius calculations. a, Spuriously labeled branch points from 26 -edge connectivity are filtered by weighting the radius of the candidate points and their neighbors. $\mathbf{b}$, Branch point filtering compared to ground-truth labeled synthetic vasculature datasets $(n$ $=20$ ). $\mathbf{c}$, Modified Euclidean distance transform $(\mathrm{mEDT}$ ) radius calculations for vessels with 1D nearest neighbors. d, Comparisons between EDT and mEDT radius calculations for an edge-vessel with singlevoxel width at numerous points. e, Comparison of the distribution of segments along 0-10 px radius bins from the glaucomatous HRF images $(n=15)$. $\mathbf{f}$, Repeated-measures analysis of the change in radius differences in the $0-5 \mathrm{px}$ radius bins from EDT to mEDT. Data are represented as mean \pm SEM. b, Data analyzed using a one-way ANOVA. e-f, Data analyzed using a repeated measures twoway ANOVA. Multiple comparisons conducted using Tukey's test. \# - Main effect of measurement method. ${ }^{* * *}-p<0.001,{ }^{* * * *}-p<0.0001$. 
A

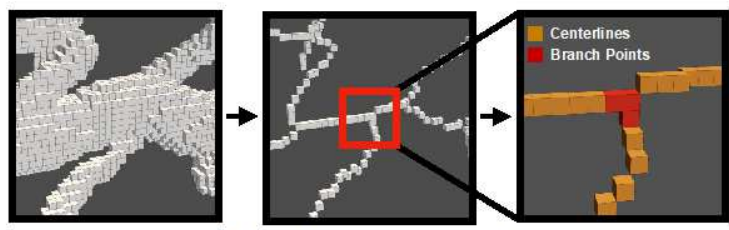

Edge detection based on 26-connectivity

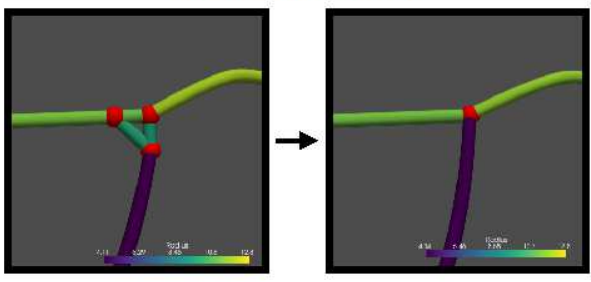

Spurious branch point removal based on parent and point radii

C
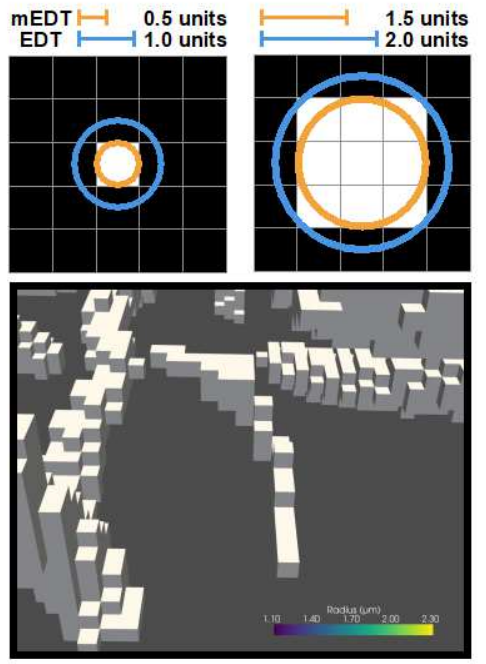

E

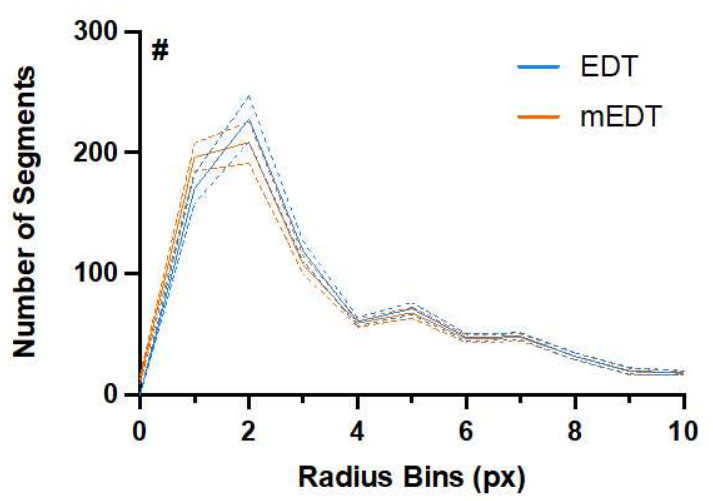

mEDT
B
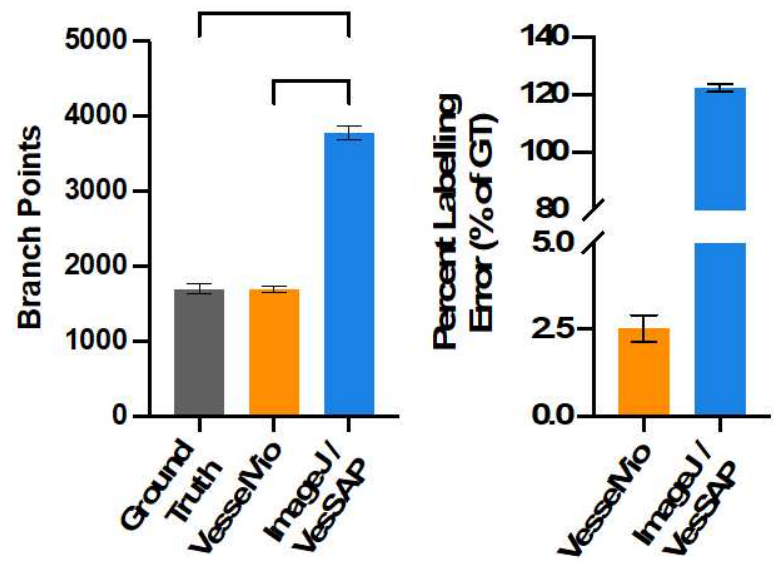

D
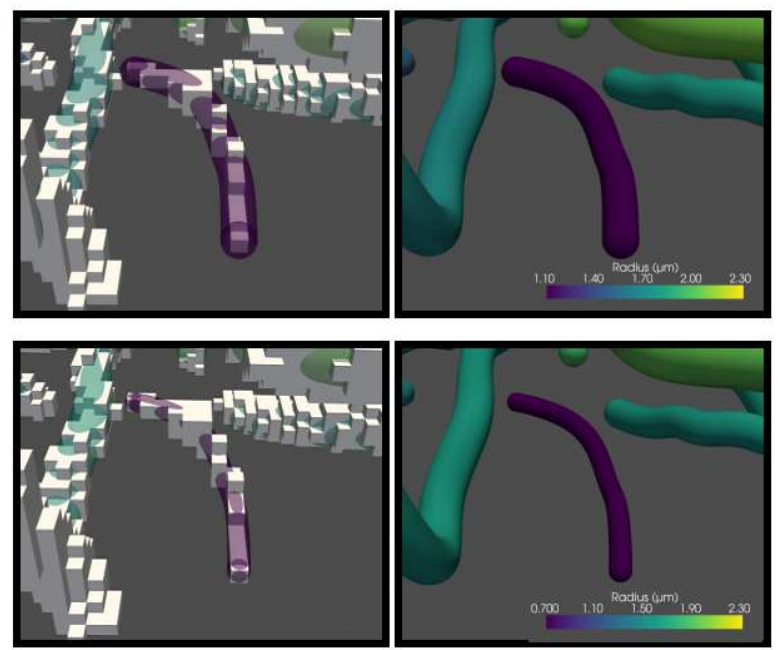

$\mathbf{F}$

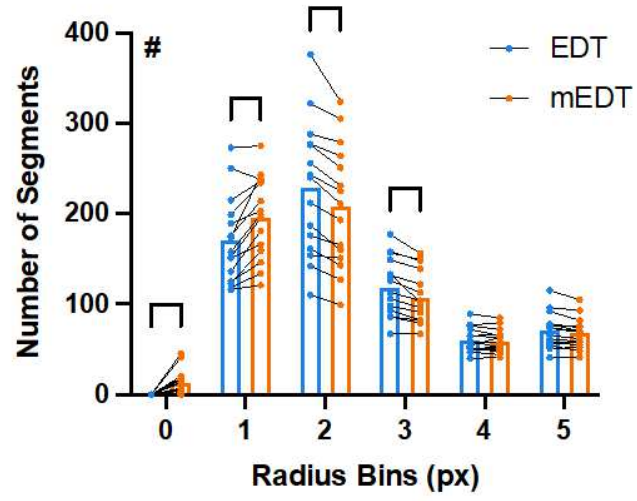




\section{Identifying Segment Radii Based on Voxel Edges}

Several techniques exist for identifying vessel radii. One technique involves recording the largest maximally inscribed spheres that can rest within mesh vessel centerline points ${ }^{29,30}$, but this technique often depends on the creation of directed graphs (i.e., manually directed vessel hierarchy) and thus was not suitable for an automated pipeline. A similar method identifies the Euclidian distance between a vessel centerline and the center of the nearest non-vessel neighbor ${ }^{6,31}$. However, one pitfall to this approach is that vessels with near-resolution or at-resolution radii are incorrectly sized when their closest non-vessel neighbor is located along the $1^{\text {st }}$ dimension of individual axes (Figure 2C), leading to oversized single-pixel/-voxel vessels (Figure 2D). This issue is not as apparent for $2^{\text {nd }}$ and $3^{\text {rd }}$ dimension neighbors (Supplementary Figure 1). As such, a simple half-unit correction for 1D neighbors (6-connectivity) is implemented to preserve small-segment radii measurements (Figure 2C). This technique enables the identification of smaller segments within vascular datasets by removing artificial inflation of segment size along the first dimension (Figure 2E, F; Supplementary Data 2).

\section{VesselVio Feature Reporting}

Following the identification of branchpoints, endpoints, and centerline radii, individual vessel segments and their features can be isolated from vascular networks. These features include average length, radius, tortuosity, surface area, and volume. Segment information can be scaled to describe network features, including network volume and skeleton length, vessel segment partitioning, segment counts, and averages of segment features. Implementation of graph theory and graph file exports from VesselVio enables users to independently identify relevant network characterization metrics, such as cohesion, network diameter, or clustering coefficient measurements ${ }^{18}$. Lastly, by leveraging the same 
back-end analysis pipeline with an additional mesh-construction stage, VesselVio can render vascular datasets for visualization and inspection of result accuracy (Figure 1C).

\section{VesselVio Quantification and Visualization}

\section{$\mu C T$, LSM, and MRI Datasets}

To demonstrate VesselVio 3D compatibility, several datasets were analyzed. First, cerebrovascular resin casts of female and male CFW mice $(n=5)$ were created as described previously ${ }^{8}$. Following tissue removal and subsequent osmication, the resulting vasculature casts were imaged using a $\mu \mathrm{CT}$ scanner at an isotropic resolution of $2.7 \mu \mathrm{m}^{3}$. Next, the inferior colliculi (IC) were segmented and binarized as example datasets (Supplementary Video 1). As expected, the IC were densely vascularized with large peaks in small diameter vessels (Figure $3 \mathrm{H}$ ); VesselVio analysis revealed numerous other vascular network features (Figure 3; Supplementary Video 2). In all, there were few sex differences in IC vasculature. However, female IC vasculature had longer segment lengths on average than males (Figure 3G; Supplementary Data 3).

Several other 3D vasculature datasets of varying imaging origins were also examined. First, a lymph node microvascular network imaged with confocal laser scanning microscopy was visualized to reveal complex capillary networks (Supplementary Video 3$)^{22}$. Visualization and analysis tests were also demonstrated on sample datasets generated by LSM (Supplementary Video 3$)^{7,10}$. Lastly, to demonstrate visualization capability with large-vessel networks, open-source composite datasets of susceptibility weighted imaging (SWI) and time-of-flight magnetic resonance angiography (ToF) from 41 human subjects were utilized ${ }^{32}$. To visualize arterial and venous vasculature simultaneously, the SWI and ToF composites were thresholded, combined, and analyzed, revealing a complex cortical vascular topology (Supplementary Video 4). Together, the analysis of rodent cerebrovasculature, 

with 3D datasets generated by various imaging techniques.

A

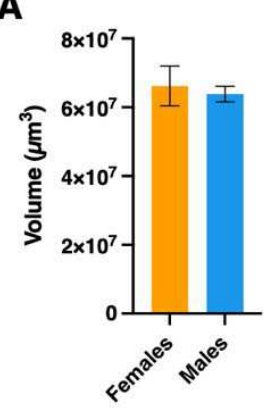

D

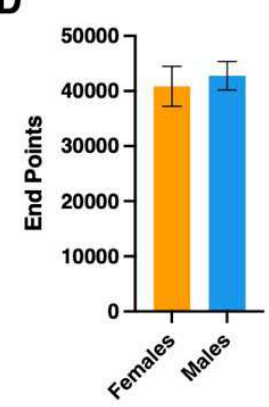

G

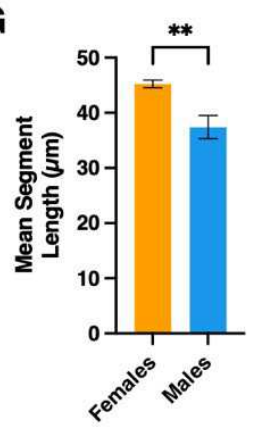

B

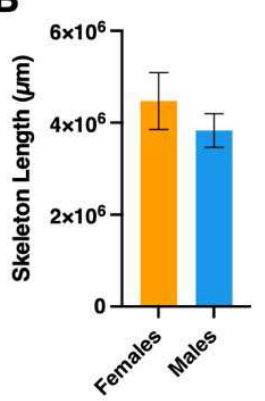

$\mathbf{E}$

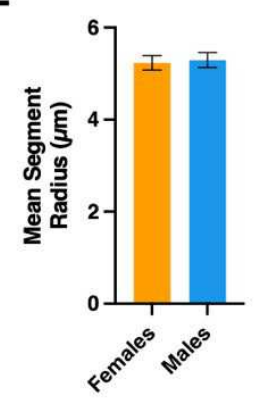

H

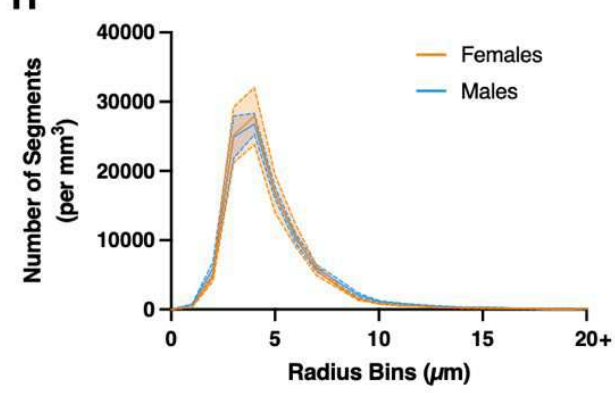

C

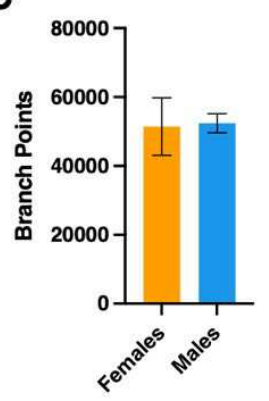

$\mathbf{F}$

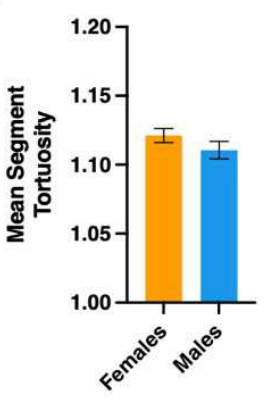

$\mathbf{J}$

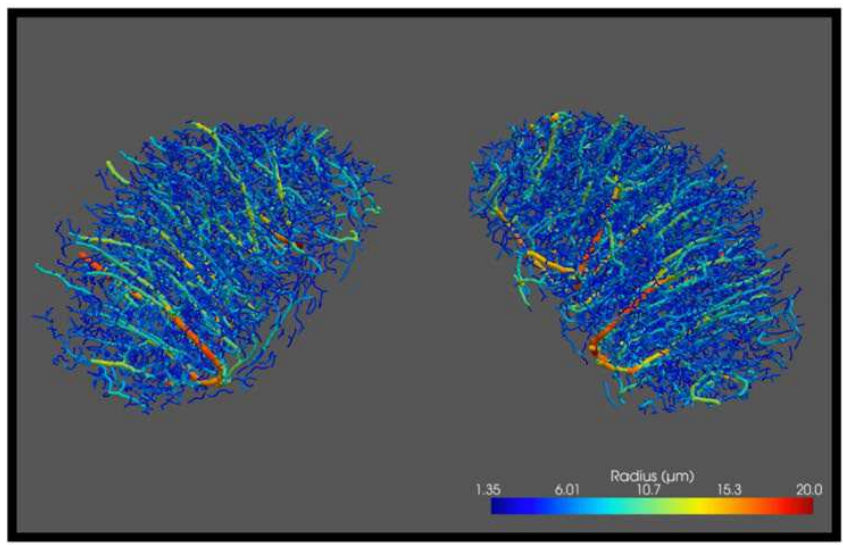

Figure 3 - Results from the analysis of inferior colliculi segmentations from female and male CFW mice. a-f, Comparisons of network area (a), length (b), branch points (c), end points (d), mean segment length $(\mathbf{e})$, mean segment tortuosity $(\mathbf{f})$, and mean segment length $(\mathbf{g})$. $\mathbf{h}$, Distribution of the number of segments along $0-20+$ radius bins. $\mathbf{i}-\mathbf{j}$, example images from female (i) and male (j) inferior colliculus reconstructions. Results for $\mathbf{a}-\mathbf{d}$ and $\mathbf{h}$ were corrected by the region-of-interest volume for individual segmentations; these results are per $\mathrm{mm}^{3}$. Data are represented as mean $\pm \mathrm{SEM}$. a-g, Data analyzed using two-tailed student's t-test. $\mathbf{h}$, Data analyzed using 2-way ANOVA; $n=5 .{ }^{* *}-p<0.01$.

\section{D Retinography}


To demonstrate the utility of VesselVio with 2D datasets, retinographs sourced from the HRF Image Database were analyzed and compared ${ }^{33}$. This database contains high-resolution images of healthy control patients, patients with diabetic retinopathy, and patients with glaucomatous eyes. VesselVio analysis revealed several differences among groups in the HRF database. Relative to healthy controls, diabetic patients present with reductions in vessel area (Figure 5A), reduced average segment radius (Figure 4A), and increased vessel tortuosity (Figure 4C). Further, relative to healthy controls, glaucomatous retinas had reduced vascular area (Figure 5A), increased branchpoints (Figure 5C), more segments (Figure 5E), and reduced average segment length (Figure 4B). Differences in the distribution of vessel radii between healthy and glaucomatous/diabetic networks occurred both in small and medium diameter vessels (Figure 5G; Supplementary Data 4).

Figure 4 - Segment characteristic comparisons among HRF groups. a, Alterations in the mean segment radius among groups. b, Differences in the mean segment length of vessels among groups. c, Alterations in the mean tortuosity of segments among the groups. Data are represented as mean \pm SEM. Data were analyzed using a one-way ANOVA; $n=15$. Upon main-effect observation, multiple comparisons were conducted using Tukey's test. ${ }^{*}-p<0.05,{ }^{* *}-p<0.01,{ }^{* * *}-p<0.001,{ }^{* * *}-p<$ 0.0001 .

Figure 5 - Results from the analysis of images from the HRF database. a-f, Comparisons of network area (a), length (b), branch points (c), end points (d), segment counts (e), and segment partitioning (f). e, Distribution of segments across $0-20+$ radius segment bins. $\mathbf{g}$, Mean length of segments in each radius bin (length in px). Data are represented as mean \pm SEM. a-f, Data analyzed using a one-way ANOVA. g-h, Data analyzed using a two-way ANOVA; $n=15$. Upon main-effect observation, multiple comparisons were conducted using Tukey's test. \# - Main effect of health condition, ${ }^{*}-p<0.05,{ }^{* *}-p$ $<0.01,{ }^{* * * *}-\mathrm{p}<0.0001$. 

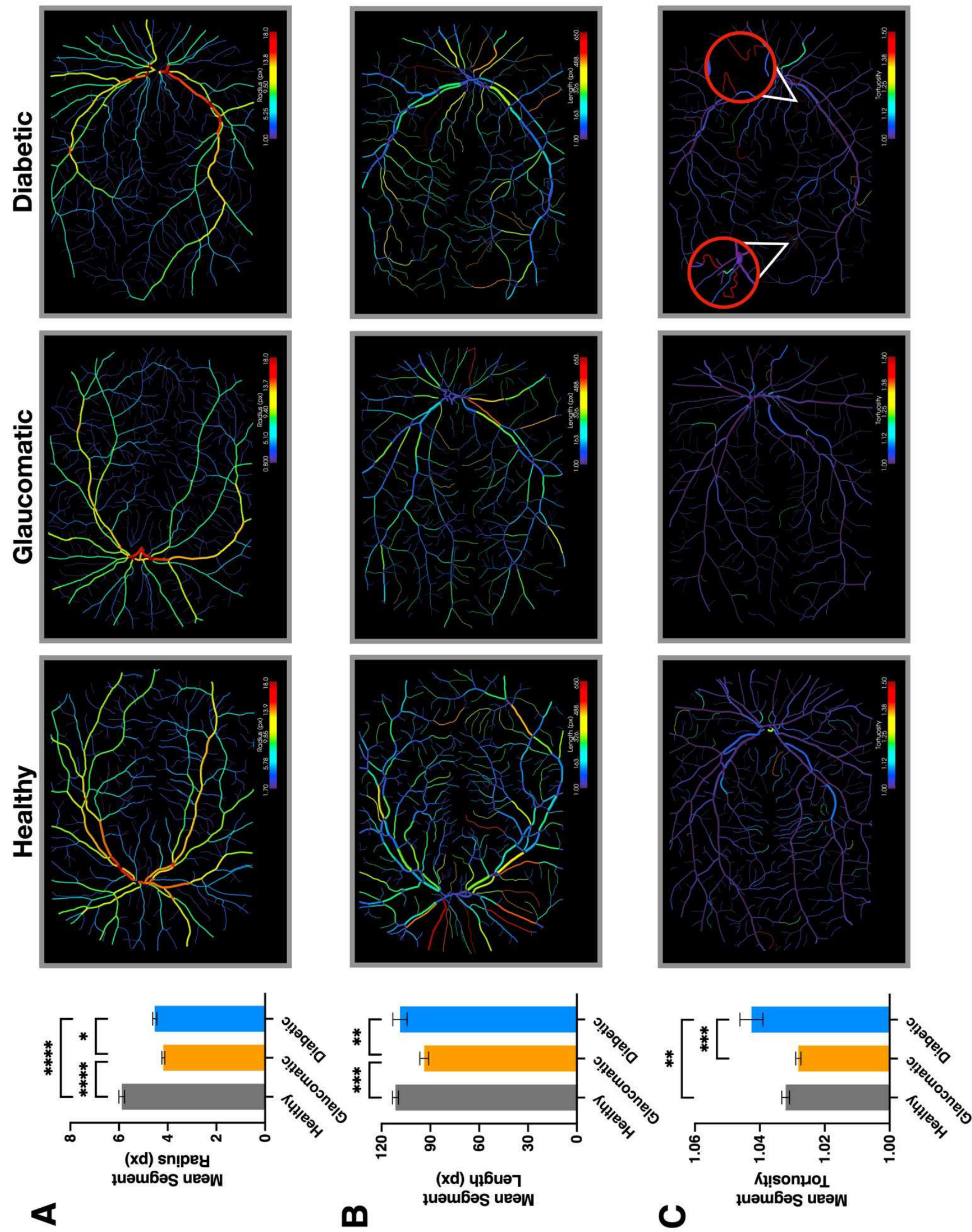
A

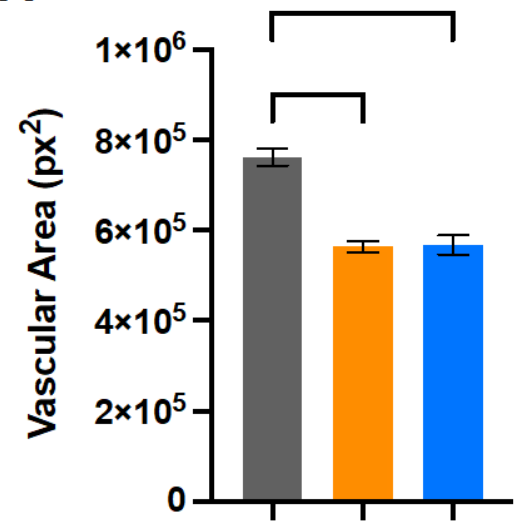

D
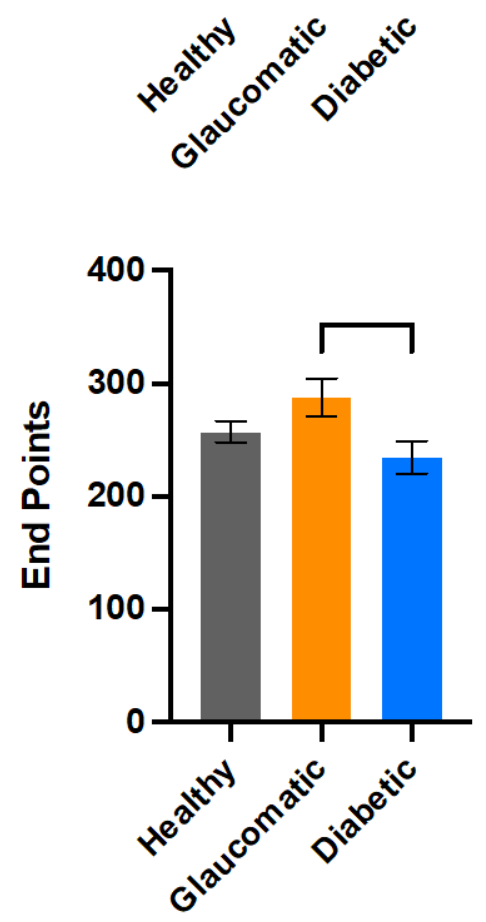

G

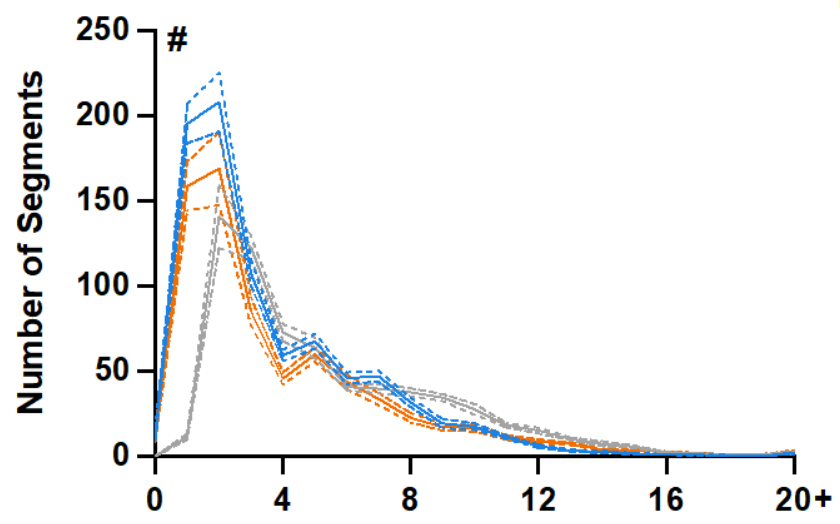

H vs. G * * * H VS. D $\sim \sim \sim \sim$ $p<0.05$

$* * * * * * *$ Radius Bins (px)
B

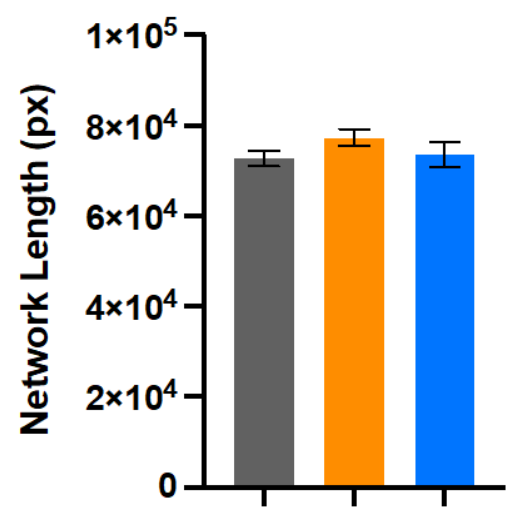

E
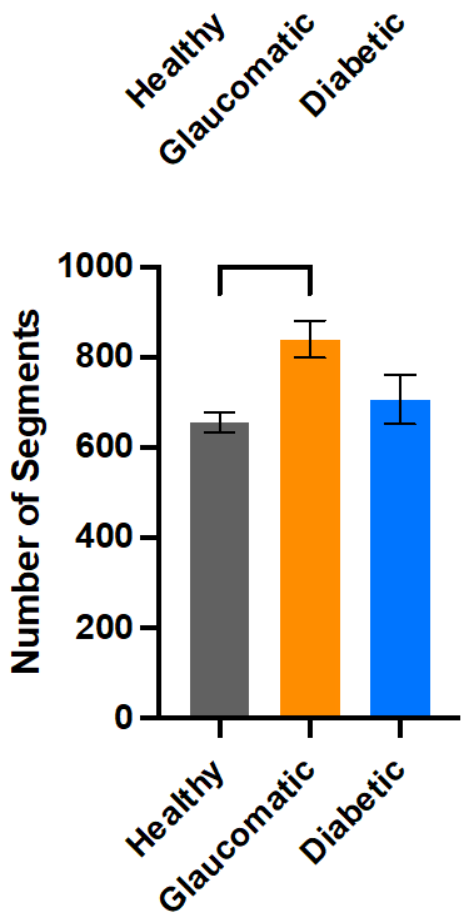

C

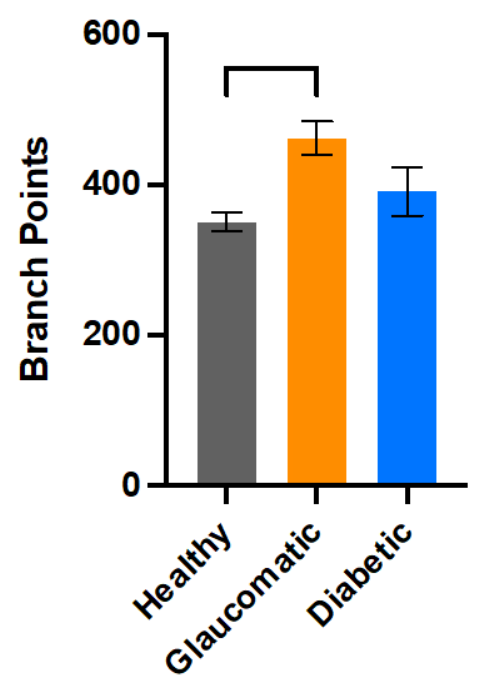

$\mathbf{F}$

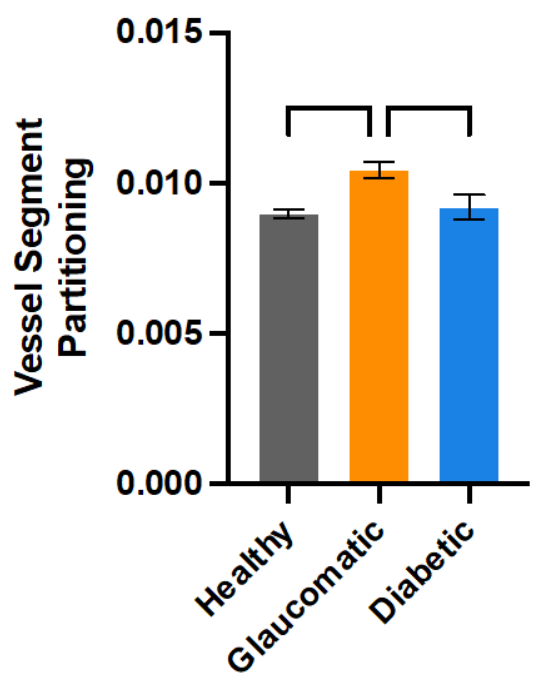

$\mathrm{H}$

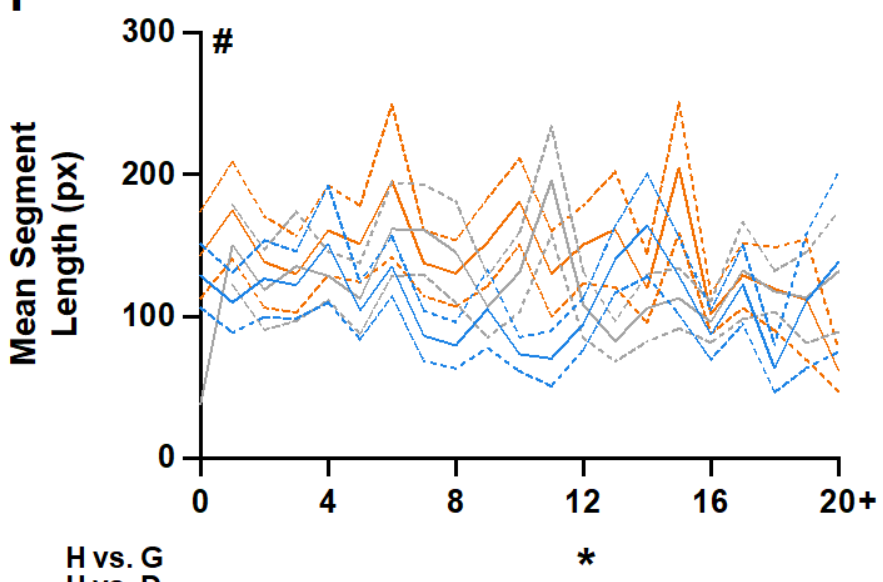

Radius Bins (px) 


\section{Discussion}

Vascular networks can dictate and indicate states of health and disease ${ }^{34}$. Characterization of the structural and community properties of these networks can improve our understanding of disease phenotypes and may lead to improved disease identification techniques and treatments. We developed an open-source application, VesselVio, for the analysis and visualization of segmented 2D and 3D vasculature datasets. Focus was centered on constructing an analysis pipeline capable of producing quantitative characterizations of numerous whole-network and small-scale segment features. Groundtruth result comparisons and two primary analyses were conducted to demonstrate the accuracy and feasibility of VesselVio for vasculature analysis.

The first set of analyses examined sex-differences in the cerebrovasculature of female and male CFW mice. There are numerous known sex-differences in the cardiovascular system, including vascular tone ${ }^{35}$, microcirculation ${ }^{36}$, and blood-brain barrier function ${ }^{37,38}$. We sought to examine how the cerebrovascular network in a specific nucleus, the inferior colliculus (IC), may differ based on sex. Analysis revealed few overall differences in the vasculature of these nuclei, save for differences in average segment lengths. However, these results still serve to demonstrate 3D network analysis utility of VesselVio.

The second set of analyses examined retinal vascular networks of healthy controls, DR patients, and patients with glaucoma. Alterations in retinal microvascular networks are associated with vision $\operatorname{loss}^{34}$, and structural changes associated with excessive or reduced angiogenesis can also serve as indicators for underlying disease states ${ }^{39,40}$. Our analyses revealed numerous vascular differences among the groups, including alterations in branchpoint density, altered vascular area, and altered tortuosity in the two disease states. Our analyses recapitulated previously reported reduced vessel area and diameter in glaucomatous eyes ${ }^{41}$, as well as increased segment tortuosity of DR eyes ${ }^{42}$. 
265 Contrasting results in DR eyes were noted compared to previous studies that reported increased vessel diameters ${ }^{43,44}$, whereas we and others observed decreased diameters ${ }^{45,46}$. However, these differences may be due to the vessel type (the HRF vessels are not separated by arterial/venous hierarchy) or vascular region analyzed. Although VesselVio is not intended to be used in any clinical diagnostic contexts, this analysis demonstrates the ability of this application to identify and characterize vascular network alterations in pathological conditions.

In common with most analytical program, the functionality of VesselVio is limited by the quality or resolution of the images that are loaded into the program. For example, if anisotropic datasets are loaded without pre-smoothing or blurring filters, then skeletonization of these datasets can produce erroneous segments. To adjust for these errors, the option to prune small, connected end-segments is included (Supplementary Video 5), but this pruning is unbiased and could hinder some types of analyses, such as angiogenesis measurements. Also, if holes or imaging artifacts are present in datasets, the skeletonization algorithm can produce unexpected results. Another limitation is that all VesselVio analyses are automated based on user-defined settings; this means the program cannot take user input for the creation of directed graphs and does not allow post-construction manual feature corrections. Although graph file export is provided, modifying the program for individual needs such as directed graph creation will require source code customization. However, open sourcing our code prioritizes transparency and customizability.

Future studies seeking to pinpoint detailed microvasculature alterations or broadly characterize regional vascular network features can employ the analytical and visualization components of VesselVio to obtain and enhance understanding of their results. In sum, VesselVio bridges the gap between modern vasculature imaging and dated analysis techniques by providing an optimized, opensource analysis pipeline and user-friendly application free for use. 


\section{Methods}

Animals

All experiments were approved by the West Virginia University Institutional Animal Care and Use Committee, and animals were maintained in accordance with NIH Animal Welfare guidelines. Adult female and male CFW mice (7- to 8-weeks of age; strain \#024; Charles River Laboratories, USA) were obtained and maintained under 14:10 light-dark cycles (lights on from 0500-1900 h). Following arrival, animals were given 1-week to acclimate to vivarium conditions before tissue collection. Food (2018 Teklad; Envigo, USA) and reverse osmosis water were provided ad libitum throughout the entire duration of the housing.

\section{Vascular Corrosion Casting and $\mu \mathrm{CT}$ Imaging}

Vascular casts of mouse brains were created using resin corrosion casting method described previously ${ }^{8}$. Perfusions occurred in the light phase between $1200-1600 \mathrm{~h}$. Prior to perfusion, mice were injected i.p. with $25 \mathrm{U}$ of heparin (63739-931-28; McKesson Corporation, USA) in $250 \mu \mathrm{L}$ of saline and then deeply anesthetized with isoflurane ( $4 \%$ induction, $1.5 \%$ maintenance) and $0.4 \mathrm{~L} /$ min oxygen flow mixture. Following confirmation of complete anesthetization, mice were perfused intracardially at a flow rate of $4 \mathrm{~mL} / \mathrm{min}$ first with $15 \mathrm{~mL}$ of $25 \mathrm{U} / \mathrm{mL}$ heparin in saline, followed by $15 \mathrm{~mL}$ of $4 \%$ paraformaldehyde (\#416780030; Acros Organics, Belgium) in saline with a pH of 7.4, followed lastly with PU4ii resin (VasQTec, Switzerland) formulated exactly as directed by the manufacturer. Five days after perfusion, the craniums were decalcified with a 12-h wash of $5 \%$ formic acid (BDH4554; VWR International, USA), the brains were dissected, and remaining tissue was removed from the casts with two 12-h washes of $7.5 \% \mathrm{KOH}\left(\mathrm{BDH} 7622\right.$; VWR International) at $35^{\circ} \mathrm{C}$. Casts were then rinsed with 
311 three 1-hour Milli-Q water washes, and the cleaned casts were osmicated in a $1 \%$ solution of osmium 312 tetroxide (\#75632; Sigma Aldrich, USA) for 12-hours to allow for optimal $x$-ray diffraction during $\mu C T$ scans. Casts were imaged on a SkyScan 1272 (Bruker, USA) at $50 \mathrm{kV} / 200 \mu \mathrm{A}$ with $360^{\circ}$ rotations in step sizes of $0.17^{\circ}$, no filter, $900 \mathrm{~ms}$ frame exposures, and 4 frame averages/step to produce an isotropic voxel resolution of $2.7 \mu \mathrm{m}^{3}$. Scan parameters were determined based on the manufacturers guidance to achieve optimal $\mathrm{x}$-ray transmission through the sample. Scans were then reconstructed using NRecon (Bruker) with beam hardening corrections at $15 \%$, ring artefacts reduction at 3 , smoothing at 0 , custom alignment compensations set for each sample, and 0.02-0.40 dynamic image ranges. Following reconstruction, the volumes were resliced coronally for inferior colliculi segmentations. Using interpolated polygonal tracing with CTAn (Bruker), inferior colliculi were manually segmented from bregma -4.9 to -5.4 using the lobule 2 of the cerebellar vermis as a landmark for bregma -4.9 (Supplementary Video 1$)^{47}$. Scans, reconstructions, re-slicing, and inferior colliculi segmentations were all conducted using the Bruker SkyScan analysis software suite.

\section{Dataset Input and Processing Preparation}

Images are loaded into VesselVio using the Simple-ITK image reader to improve compatibility with various file formats ${ }^{48}$. It is important to note that VesselVio is only compatible with vascular datasets that have been pre-segmented and binarized. To ensure that datasets loaded into the program are prepared appropriately for subsequent analysis, we re-binarize all inputs with a threshold of 1 and convert the image stack or single image into a binarized contiguous array of 0 -value background and 1-value foreground elements. If images are not loaded in 8-bit grayscale, then this binarization to unsigned integers process can save memory during subsequent processing. All array processing in VesselVio is conducted with NumPy 49 


\section{Volume Skeletonization and Centerline Extraction}

VesselVio employs the scikit-image ${ }^{50}$ implementation of a widely used medial axis parallel thinning algorithm ${ }^{25}$. We selected this algorithm because it produces few erroneous branchpoint extensions, particularly when used with high-resolution datasets. This algorithm is also capable of thinning 2D and 3D datasets, making it optimal for analytical purposes. Following skeletonization, (n, $3)$ or $(n, 2)$ arrays are created containing Cartesian coordinate information of the location of all vessel centerlines.

\section{Radii Calculations}

Before the creation of the undirected graph, vessel centerline radii measurements are conducted. Previous publications focused on voxel/pixel vasculature analysis have utilized Euclidean distance transforms (EDT) on segmented vasculature images to find centerline radii ${ }^{7,51}$. These blanketed EDT methods find the Euclidean distance (ED) from the centerline point to the center of the nearest non-vessel neighbor point. However, an apparent unconsidered flaw in this traditional method is that finding the ED to the nearest non-vessel neighbor can overestimate the radius of the vessel if it is measured in single-dimension orientations from the centerline vessel. For example, given an isotropic

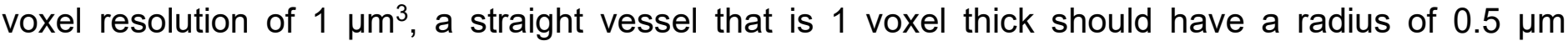
(Supplementary Figure 1). However, the traditional EDT method will record this vessel as having a radius of $1 \mu \mathrm{m}^{3}$, doubling the apparent diameter. This is because ED measurements are calculated between the coordinates of the center of the centerline voxel and the center of its non-vessel neighbor. Radii inflations in traditional EDT measurements are less pronounced as vessels become larger, but small-diameter vessels can be binned incorrectly using this blanketed technique. Instead, the 
calculation should be made to the edge of the nearest non-voxel neighbor if it is oriented singledimensionally to the centerline point. Because of this, a modified EDT (mEDT) calculation was implemented that has 0.5 -unit corrections for all neighbors oriented along single $\mathrm{X}, \mathrm{Y}$, or $\mathrm{Z}$ axis directions.

An argument could be made to calculate distance to the corner of the center pixel/voxel, but the edge was selected. Given the phenotypical tubular shape of vessels, EDT-based radii measurements are more appropriate for neighbors located along two- and three-dimensional orientations in 2D or 3D space. Thus, only radii measurements for nearest neighbors in 1D space are corrected (Supplementary Figure 1).

In previous publications, EDTs are applied to the entire image, and the centerline values are then extracted. However, this process is computationally expensive and leads to difficulty with making the intended 1D corrections. As such, the mEDT method only calculates the ED of centerline points to the nearest non-vessel neighbors, rather than transforming the entire image. Points in the previously constructed centerline coordinate arrays are used as seeds for the placement of expanding boxes that search centerline point neighborhoods for non-vessel neighbors. This box expands around a centerline point until at least four non-vessel neighbors are identified, rather than a single neighbor as with previous implementations. Then, rather than directly computing the ED (square root of sum of squared deltas) between the centerline point and neighbor (1), the user finds the absolute deltas of the centerline and neighbor coordinates and loads the deltas into an mEDT lookup table with precomputed and precorrected ED radius values (2). This lookup table is constructed to a user-specified maximum size and has half-pixel/-voxel unit corrections for all measurements along single $\mathrm{X}, \mathrm{Y}$, or $\mathrm{Z}$ axes (e.g., a delta array of $[0,0,5]$ returns a 4.5 voxel distance). 


$$
d_{\text {radius }}(c, b)=\sqrt{\sum_{i=1}^{n}\left(c_{i}-b_{i}\right)^{2}}
$$

$$
d_{\text {radius }}=m E D T_{L U T}\left[\left|c_{x}-b_{x}\right|,\left|c_{y}-b_{y}\right|,\left|c_{z}-b_{z}\right|\right]
$$

After the distance for each non-vessel neighbor is calculated, the four lowest values are averaged, and this average is defined as the radius for the centerline point. Four values are averaged to improve the accuracy of radii measurements and account for potential small vessel surface divots, bubbles, or imaging artifacts common in various vascular preparation techniques, such as corrosion casting. This process is repeated for all centerline points. Usage of just-in-time compilation and parallel processing with Numba ${ }^{52}$ makes this centerline-targeted mEDT process effective and rapid in comparison to previous whole-image EDT approaches. Future work seeking to implement this centerline-specific EDT method can easily access or modify our source code for other needs.

\section{Graph Construction}

Following radii measurements, VesselVio constructs undirected graphs to represent the vasculature skeleton in an abstract space. To do this, the number of centerline points is identified, and an equal number of isolated vertices are added to the graph. Centerline points are assigned a vertex index based on their order of appearance in our point Cartesian coordinate array; these indices are then stored in a temporary lookup table used for edge creation in the graph. Further, each vertex is given a coordinate and radius attribute based on its corresponding point values. Next, the forward facing 13-connectivity neighborhood of all centerline points is scanned to identify immediate neighbors. Upon neighbor identification, an edge is created between the vertices. Unidirectional scanning in edge identification process prevents parallel edges from being created 6 . 
Following graph creation, $\mathrm{n} !=2$ degree vertices are filtered from the graph. The remaining components are then scanned, and isolated segments and endpoint segments shorter than userdefined sizes are pruned (Supplementary Video 5). Following the removal of isolated segments from the graph, a custom flood-filling process is implemented to remove corresponding pixels/voxels from the original volume. Flood-filling of these isolated segments prevents inflation of the calculated area/volume. This technique is not implemented for endpoint segments, as endpoint segment pruning is used to remove erroneous centerlines created during skeletonization. From our tests, endpoint segments in need of pruning are often incorrectly skeletonized centerlines within large segments, so flood filling is not implemented for these segments.

Graphical representation of the vasculature construction also allows for improved identification of branchpoints. Branchpoints are often identified based on simple neighborhood connectivity; if a centerline point has more than two neighbors, it is defined as a branchpoint ${ }^{7,16}$. However, this method leads to an artificial inflation of identified branchpoints; this is because at branchpoint junctions, multiple vertices can have $>2$ neighbors - not just a single branchpoint. In graphical space, these falsely identified branchpoints form small loops, or cliques. To correct for this, we constructed a set of 12 branchpoint filtering algorithms that remove $>99 \%$ of identified branchpoint cliques as determined by the datasets analyzed in this manuscript (Supplementary Data 1; Supplementary Video 6). Briefly, branchpoints are isolated from the main graph, and identified cliques are individually processed. A clique is classified based on its number of edges and branchpoints, and the appropriate filter is applied to identify the remaining branchpoint(s). Candidate branchpoints in the clique are positively weighted based on their radius, the radius of their neighbors, the degree of their connectivity. The highest weighted candidate(s) is/are then labelled as branchpoints, and the selected filter removes appropriate edges within the clique to eliminate branchpoint status of the lowest weighted candidate(s). The 
pseudocode for these algorithms is in Supplementary text (Supplementary Notes), and all original code can be found at our GitHub page (https://github.com/JacobBumgarner/VesselVio).

Aside from area/volume measurements, all further individual segment characteristics and wholenetwork features are extracted from the constructed graph. Graph creation and processing in VesselVio uses the python igraph package ${ }^{53}$.

\section{Feature Extraction}

Volume/area are measured by finding the number of voxels/pixels in the image after segment filtering. Branchpoint and endpoint counts are determined by the number of $n>3$ and $n=1$ degree vertices in the graph, respectively. Segments are identified in the graph by filtering $n>2$ degree vertices and then sorting through the remaining individual components. After each segment is identified, a mean segment radius is determined from the averaged vertices that construct the segment. Because calculating segment length based on vertex-vertex edges produces paths that are irregular to the vessel surface, a smoother path is constructed by creating a cubic B-spline from the coordinates of the original segment vertices using the geomdl package ${ }^{54}$. Quadratic B-splines are created for segments with 3 coordinate points. The length along the spline is then approximated using EDTs between a defined number of points along the spline identified using the Cox-De Boor algorithm ${ }^{54}$. Then, tortuosity measurements are created by finding the arc-cord ratio of the segment (segment length divided by the ED between the start and end points). Averages of segments are then created and automatically binned for ease of analysis. Segment partitioning is also calculated, which is the inverse of average segment length calculated by dividing the number of segments by the total network length ${ }^{55}$.

\section{Mesh Visualization and Application Interface}


To construct meshes for visualization, we leverage the high-level python package PyVista ${ }^{56}$ that $^{2}$

wraps The Visualization Toolkit. We create individual poly-datasets from our segment splines, apply tube filters to create simple network and scaled network (based on average radius) meshes, and assign each tube a radius, length, and tortuosity scalar for visualization. All scalars and scaled segment sizes are based on the mean of segment features. These segments are combined into an undirected grid for surface extraction and subsequent rendering. Additional branchpoint and endpoint meshes are created. Then, original (voxel/pixel based) and smoothed surface meshes (marching cubes based-based) are created from the filtered input volume so researchers can visually validate the output features from the program by comparing simple/scaled networks to the original/smoothed meshes. Lastly, construction of the front-end application for VesselVio was accomplished with PyQt5 under GNU GPLv3 licensing (Supplementary Figure 2).

\section{Statistical Analysis}

All statistical analyses were conducted using Prism 9 (GraphPad; USA). Synthetic vasculature results were analyzed using one-way ANOVA tests and repeated-measures two-way ANOVA tests. HRF datasets were analyzed using one-way ANOVA tests. Mice IC data were analyzed using twotailed student's t-tests. Distributions of segment counts per radii bin, average segment length per radii bin, and segment tortuosity per radii bin were all analyzed using two-way ANOVA tests. Following main effects observations in one- or two-way ANOVA tests, multiple comparisons were made using Tukey's test. An alpha of 0.05 was set for statistical significance.

\section{Data Availability}


The synthetic vasculature datasets and inferior colliculi segmentations analyzed in this article are available at the VesselVio Repository on the Harvard Dataverse website (https://dataverse.harvard.edu/dataverse/VesselVio-Datasets). All data generated in this manuscript are in the Supplementary files.

\section{Code Availability}

Original and updated source-code for VesselVio are available on GitHub (https://github.com/JacobBumgarner/VesselVio). Downloads for the MacOS and Windows standalone application can be found at the VesselVio homepage (https://jacobbumgarner.github.io/VesselVio/). Pseudocode for the branch point filtering algorithms is in the Supplementary Notes.

\section{Acknowledgements}

We acknowledge R.C. White for his assistance with corrosion cast generation and manuscript proofing. This project was supported by National Institute of Health grant R01NS092388.

\section{Author Contributions}

J.R.B. wrote the program source code, collected data, and analyzed data, J.R.B. and R.J.N. conceived the program, reviewed data analyses, and wrote and edited the manuscript.

\section{Competing Interests Statement}

The authors declare no competing interests. 


\section{References}

1. Teuwen L-A, Geldhof V, Pasut A, Carmeliet P. Covid-19: The vasculature unleashed. Nat. Rev. Immunol. 20, 389-91 (2020).

2. Durham JT, Herman IM. Microvascular modifications in diabetic retinopathy. Curr. Diabetes Rep. 11, 253-64 (2011).

3. Jiang X, Andjelkovic AV, Zhu L, Yang T, Bennett MVL, Chen J, Keep RF, Shi Y. Blood-brain barrier dysfunction and recovery after ischemic stroke. Prog. Neurobiol. 163, 144-71 (2018).

4. Govindpani K, McNamara LG, Smith NR, Vinnakota C, Waldvogel HJ, Faull RLM, Kwakowsky A. Vascular dysfunction in alzheimer's disease: A prelude to the pathological process or a consequence of it. J. Clin. Med. 8, 651 (2019).

5. Di Giovanna AP, Tibo A, Silvestri L, Müllenbroich MC, Costantini I, Mascaro ALA, Sacconi L, Frasconi P, Pavone FS. Whole-brain vasculature reconstruction at the single capillary level. Sci. Rep. 8, 1-11 (2018).

6. Kirst C, Skriabine S, Vieites-Prado A, Topilko T, Bertin P, Gerschenfeld G, Verny F, Topilko P, Michalski N, Tessier-Lavigne M. Mapping the fine-scale organization and plasticity of the brain vasculature. Cell. 180, 780-795. e25 (2020).

7. Todorov MI, Paetzold JC, Schoppe O, Tetteh G, Shit S, Efremov V, Todorov-Völgyi K, Düring M, Dichgans M, Piraud M. Machine learning analysis of whole mouse brain vasculature. Nat. Methods. 17, 442-9 (2020).

8. Quintana DD, Lewis SE, Anantula Y, Garcia JA, Sarkar SN, Cavendish JZ, Brown CM, Simpkins JW. The cerebral angiome: High resolution microct imaging of the whole brain cerebrovasculature in female and male mice. Neurolmage. 202, 116109 (2019).

9. Schaad L, Hlushchuk R, Barré S, Gianni-Barrera R, Haberthür D, Banfi A, Djonov V. Correlative imaging of the murine hind limb vasculature and muscle tissue by microct and light microscopy. Sci. Rep. 7, 1-12 (2017).

10. Haft-Javaherian M, Fang L, Muse V, Schaffer CB, Nishimura N, Sabuncu MR. Deep convolutional neural networks for segmenting $3 d$ in vivo multiphoton images of vasculature in alzheimer disease mouse models. PLoS One. 14, e0213539 (2019).

11. Zudaire E, Gambardella L, Kurcz C, Vermeren S. A computational tool for quantitative analysis of vascular networks. PLoS One. 6, e27385 (2011).

12. Niemisto A, Dunmire V, Yli-Harja O, Zhang W, Shmulevich I. Robust quantification of in vitro angiogenesis through image analysis. IEEE Trans. Med. Imag. 24, 549-53 (2005).

13. Mazzaferri J, Larrivée B, Cakir B, Sapieha P, Costantino S. A machine learning approach for automated assessment of retinal vasculature in the oxygen induced retinopathy model. Sci. Rep. 8, 1-11 (2018).

14. Montoya-Zegarra JA, Russo E, Runge P, Jadhav M, Willrodt A-H, Stoma S, Nørrelykke SF, Detmar M, Halin C. Autotube: A novel software for the automated morphometric analysis of vascular networks in tissues. Angiogenesis. 22, 223-36 (2019).

15. Rust R, Kirabali T, Grönnert L, Dogancay B, Limasale YDP, Meinhardt A, Werner C, Laviña B, Kulic L, Nitsch RM. A practical guide to the automated analysis of vascular growth, maturation and injury in the brain. Front. Neurosci. 14, 244 (2020).

16. Arganda-Carreras I, Fernández-González R, Muñoz-Barrutia A, Ortiz-De-Solorzano C. 3d reconstruction of histological sections: Application to mammary gland tissue. Microsc. Res. Tech. 73, 1019-29 (2010). 
17. Tetteh G, Efremov V, Forkert ND, Schneider M, Kirschke J, Weber B, Zimmer C, Piraud M, Menze BH. Deepvesselnet: Vessel segmentation, centerline prediction, and bifurcation detection in 3-d angiographic volumes. Front. Neurosci. 14, (2020).

18. Hahn A, Bode J, Krüwel T, Solecki G, Heiland S, Bendszus M, Tews B, Winkler F, Breckwoldt $\mathrm{MO}$, Kurz FT. Glioblastoma multiforme restructures the topological connectivity of cerebrovascular networks. Sci. Rep. 9, 1-17 (2019).

19. Chapman BE, Berty HP, Schulthies SL. Automated generation of directed graphs from vascular segmentations. J. Biomed. Inform. 56, 395-405 (2015).

20. Jafarnejad M, Ismail AZ, Duarte D, Vyas C, Ghahramani A, Zawieja DC, Celso CL, Poologasundarampillai G, Moore JE. Quantification of the whole lymph node vasculature based on tomography of the vessel corrosion casts. Sci. Rep. 9, 1-11 (2019).

21. Epah J, Pálfi K, Dienst FL, Malacarne PF, Bremer R, Salamon M, Kumar S, Jo H, Schürmann C, Brandes RP. 3d imaging and quantitative analysis of vascular networks: A comparison of ultramicroscopy and micro-computed tomography. Theranostics. 8, 2117 (2018).

22. Kelch ID, Bogle G, Sands GB, Phillips ARJ, LeGrice IJ, Dunbar PR. Organ-wide 3d-imaging and topological analysis of the continuous microvascular network in a murine lymph node. Sci. Rep. 5, 1-19 (2015).

23. Selle D, Preim B, Schenk A, Peitgen $\mathrm{H}-\mathrm{O}$. Analysis of vasculature for liver surgical planning. IEEE Trans. Med. Imag. 21, 1344-57 (2002).

24. Czech W, Dzwinel W, Arodz T, Dudek AZ. Exploring complex networks with graph investigator research application. Comput. Inform. 30, 381-410 (2011).

25. Lee T-C, Kashyap RL, Chu C-N. Building skeleton models via 3-d medial surface axis thinning algorithms. CVGIP - Graph. Model. Im. 56, 462-78 (1994).

26. Palágyi K, Tschirren J, Hoffman EA, Sonka M. Quantitative analysis of pulmonary airway tree structures. Comput. Biol. Med. 36, 974-96 (2006).

27. Chen Y, Laura CO, Drechsler K. Generation of a graph representation from three-dimensional skeletons of the liver vasculature. 2009 2nd International Conference on Biomedical Engineering and Informatics. 1-5 (2009).

28. Tetteh G, Efremov V, Forkert ND, Schneider M, Kirschke J, Weber B, Zimmer C, Piraud M, Menze BH. Deepvesselnet: Vessel segmentation, centerline prediction, and bifurcation detection in 3-d angiographic volumes. Front. Neurosci. 14, (2020).

29. Antiga L, Ene-lordache B, Remuzzi A. Computational geometry for patient-specific reconstruction and meshing of blood vessels from $\mathrm{mr}$ and ct angiography. IEEE Trans. Med. Imag. 22, 674-84 (2003).

30. Antiga L, Steinman DA. Robust and objective decomposition and mapping of bifurcating vessels. IEEE Trans. Med. Imag. 23, 704-13 (2004).

31. Mouches P, Forkert ND. A statistical atlas of cerebral arteries generated using multi-center mra datasets from healthy subjects. Sci. Data. 6, 1-8 (2019).

32. Bernier M, Cunnane SC, Whittingstall $\mathrm{K}$. The morphology of the human cerebrovascular system. Hum. Brain Mapp. 39, $4962-75$ (2018).

33. Budai A, Bock R, Maier A, Hornegger J, Michelson G. Robust vessel segmentation in fundus images. Int. J. Biomed. Imaging. 2013, (2013).

34. Carmeliet P. Angiogenesis in life, disease and medicine. Nature. 438, 932-6 (2005). 
35. Boese AC, Kim SC, Yin K-J, Lee J-P, Hamblin MH. Sex differences in vascular physiology and pathophysiology: Estrogen and androgen signaling in health and disease. Am. J. Physiol. Heart Circ. 313, H524-45 (2017).

36. Huxley VH, Kemp SS in Sex-Specific Analysis Of Cardiovascular Function (eds Kerkhof PLM, Miller VM) 307-28 (Springer, New York City, 2018).

37. Robison LS, Gannon OJ, Salinero AE, Zuloaga KL. Contributions of sex to cerebrovascular function and pathology. Brain Res. 1710, 43-60 (2019).

38. Humphries KH, Izadnegadar M, Sedlak T, Saw J, Johnston N, Schenck-Gustafsson K, Shah RU, Regitz-Zagrosek V, Grewal J, Vaccarino V. Sex differences in cardiovascular disease-impact on care and outcomes. Front. Neuroendocrinol. 46, 46 (2017).

39. Lee R, Wong TY, Sabanayagam C. Epidemiology of diabetic retinopathy, diabetic macular edema and related vision loss. Eye Vis. 2, 1-25 (2015).

40. Selvam S, Kumar T, Fruttiger M. Retinal vasculature development in health and disease. Prog. Retin. Eye Res. 63, 1-19 (2018).

41. Chan KKW, Tang $\mathrm{F}$, Tham CCY, Young AL, Cheung CY. Retinal vasculature in glaucoma: $A$ review. BMJ Open Ophthalmol. 1, e000032 (2017).

42. Sasongko MB, Wong TY, Nguyen TT, Cheung CY, Shaw JE, Wang JJ. Retinal vascular tortuosity in persons with diabetes and diabetic retinopathy. Diabetologia. 54, 2409-16 (2011).

43. Bek T. Diameter changes of retinal vessels in diabetic retinopathy. Curr. Diabetes Rep. 17, 1-7 (2017).

44. Klein R, Myers CE, Lee KE, Gangnon R, Klein BEK. Changes in retinal vessel diameter and incidence and progression of diabetic retinopathy. Arch. Ophthalmol. 130, 749-55 (2012).

45. Adhi M, Brewer E, Waheed NK, Duker JS. Analysis of morphological features and vascular layers of choroid in diabetic retinopathy using spectral-domain optical coherence tomography. JAMA Ophthalmol. 131, 1267-74 (2013).

46. Fryczkowski AW, Sato SE, Hodes BL. Changes in the diabetic choroidal vasculature: Scanning electron microscopy findings. Ann. Ophthalmol. 20, 299-305 (1988).

47. Paxinos G, Franklin KBJ. Paxinos and Franklin's the mouse brain in stereotaxic coordinates (Academic Press, Cambridge, 2019).

48. Lowekamp BC, Chen DT, Ibáñez L, Blezek D. The design of simpleitk. Front. Neuroinform. 7, 45 (2013).

49. Harris CR, Millman KJ, van der Walt SJ, Gommers R, Virtanen P, Cournapeau D, Wieser E, Taylor J, Berg S, Smith NJ. Array programming with numpy. Nature. 585, 357-62 (2020).

50. Van der Walt S, Schönberger JL, Nunez-Iglesias J, Boulogne F, Warner JD, Yager N, Gouillart E, Yu T. Scikit-image: Image processing in python. PeerJ. 2, e453 (2014).

51. Tsai PS, Kaufhold JP, Blinder P, Friedman B, Drew PJ, Karten HJ, Lyden PD, Kleinfeld D. Correlations of neuronal and microvascular densities in murine cortex revealed by direct counting and colocalization of nuclei and vessels. J. Neurosci. 29, 14553-70 (2009).

52. Lam SK, Pitrou A, Seibert S. Numba: A Ilvm-based python jit compiler. Proceedings of the Second Workshop on the LLVM Compiler Infrastructure in HPC. 7, 1-6 (2015).

53. Csardi G, Nepusz T. The igraph software package for complex network research. Int. J. Complex Syst. 1695, 1-9 (2006).

54. Bingol OR, Krishnamurthy A. Nurbs-python: An open-source object-oriented nurbs modeling framework in python. SoftwareX. 9, 85-94 (2019). 
55. Corliss BA, Mathews C, Doty R, Rohde G, Peirce SM. Methods to label, image, and analyze the complex structural architectures of microvascular networks. Microcirculation. 26, e12520 (2019).

56. Sullivan CB, Kaszynski AA. Pyvista: $3 d$ plotting and mesh analysis through a streamlined interface for the visualization toolkit (vtk). J. Open Source Softw. 4, 1450 (2019). 


\section{Supplementary Files}

This is a list of supplementary files associated with this preprint. Click to download.

- SupplementaryNotes.docx

- SupplementaryData1CliqueFilteringResults.xIsx

- SupplementaryData2mEDTvsEDTDistributionResults.xlsx

- SupplementaryData3ICResults.xIsx

- SupplementaryData4HRFResults.xIsx

- SupplementaryFigure1EDTvsmEDT.docx

- SupplementaryFigure2ApplicationImages.docx

- SupplementaryVideo1ICSegmentation.mov

- SupplementaryVideo2InferiorColliculusOrbits.mov

- SupplementaryVideo3LSMDatasets.mov

- SupplementaryVideo4LeftHemisphereofToFSWIComposite.mov

- SupplementaryVideo5BranchPruning.mov

- SupplementaryVideo6BranchPointFiltering.mov 